\title{
On the Distribution of Parity in the Partition Function
}

\section{By Thomas R. Parkin and Daniel Shanks}

1. Introduction. Let $p(n)$ be the number of (unrestricted) partitions of $n$, and define $p(0)=1$. Then $p(n)$ is generated by

$$
\sum_{n=0}^{\infty} p(n) x^{n}=\prod_{n=1}^{\infty} \frac{1}{\left(1-x^{n}\right)}=\left\{1+\sum_{n=1}^{\infty}(-1)^{n}\left[x^{n(3 n-1) / 2}+x^{n(3 n+1) / 2}\right]\right\}^{-1} .
$$

There is little known about $p(n)$ modulo 2 ; in particular, there are no known criteria for the parity of $p(n)$ comparable in simplicity with Ramanujan's famous sufficient condition for divisibility by 5 :

$$
5 \mid p(5 k+4) \text {. }
$$

Kolberg [1] proved, but by contradiction and without identifying the arguments $n$, that i vitely many $p(n)$ are even, and infinitely many are odd. His proof is almost as simple as Euclid's proof that there are infinitely many primes, but like that proof it offers only very little more in the way of exact information concerning questions of distribution.

From Gupta's tables [2], [3] we find the following cumulative distribution into odds and evens for $0 \leqq n \leqq 499$.

\begin{tabular}{c|cc|c|c|c} 
& $n \leqq 99$ & $n \leqq 199$ & $n \leqq 299$ & $n \leqq 399$ & $n \leqq 499$ \\
\hline ()dds & 58 & 111 & 171 & 222 & 277 \\
\hline livens & 42 & 89 & 129 & 178 & 223 \\
\hline
\end{tabular}

In the absence of any known reason to the contrary, and because of the rather unsmooth recursion for $p(n)$ implied by (1), it would be natural to guess that the evens and odds are equinumerous, i.e., that the ratio of their counts has the limit 1 as the upper bound for $n \rightarrow \infty$. But the early preponderance of the odds, as just tabulated, would make us hesitate to conjecture that this is true. Nonetheless, it seemed to us not unlikely that this early preponderance might wash out as later returns came in (from upstate, so to speak). But it does seem unlikely that a theoretical proof of this could be attained with known techniques.

We have therefore examined the question empirically with a computer, and have put an even stronger question. Consider the number $m=1.74264258 \cdots$, which when written in binary:

$$
m=1.10111110000111011101 \cdots,
$$

has its $k$ th bit to the right of the binary point 0 or 1 according as $p(k)$ is even or odd. ( $m$ stands for Major MacMahon.) We now ask if $m$ is normal with respect to the base 2 . If so, this not only implies the previously supposed equinumerosity, but

Received February 13, 1967. 
also implies that all possible pairs, 00,01, 10, and 11, have an asymptotic density of $\frac{1}{4}$, etc.

Here, however, we must note that the corresponding proposition modulo 5 is definitely false. Thus, if

$$
r=1.12302102021210112002 \cdots
$$

is a number written in quinary with its $k$ th place $\equiv p(k)$ modulo 5 , we know from (2) that $r$ is not normal. In fact, $r$ is not even simply normal since it is further known that more than $20 \%$ of the $p(n)$ are divisible by 5 . For, in addition to (2), Morris Newman shows, in the following paper [4], that

$$
5 \mid p\left(5 \cdot 19^{4} k+15147\right)
$$

also, and still other independent linear functions also have this property. (A. O. L. Atkin has obtained more general results; these will appear in [5].)

One of our reasons for stressing this failure modulo 5 is because of the character of our main problem. Suppose, for instance, that our empirical investigation shows that parity does appear to be equinumerous, and even normal. Then one might well remark: "So what? Isn't that what one expects?" But the failure modulo 5 puts the problem in a more interesting light.

We have determined the parity of $p(n)$ up to $n=2,039,999$. In what follows we will indicate our method, our results, and some related investigations.

2. Notation and Nomenclature. Let $a_{n}$ be the $n$th bit of $m$ in (3):

$$
a_{n} \equiv p(n) \quad(\bmod 2) .
$$

Let the finite sequence

$$
a_{m} a_{m+1} a_{m+2} \cdots a_{m+k-1}
$$

be called the $m$ th $k$-tuple. Thus 1101 is the 0 th 4 -tuple and 11111 is the 3 rd 5 -tuple. There are $2^{k}$ possible types of $k$-tuple, and let us designate these $2^{k}$ types by the integer, which, when written in binary, is the $k$-tuple itself. Thus 1101 is the 13th type of 4-tuple and 11111 is the 31st type of 5-tuple. Let

$$
\sum_{t}^{(k)}(n)
$$

be the number of $t$ type $k$-tuples that appear to the left of, but not including, the $n$th $k$-tuple. (We find it convenient, because of (11) below, to count the 0 th $k$-tuple here, and therefore to omit the $n$ th, so that the argument $n$ in $\sum_{t}^{(k)}(n)$ means that $n k$-tuples have been counted.) Thus, from (3),

$$
\sum_{0}^{(2)}(10)=2, \quad \sum_{1}^{(2)}(10)=1, \quad \sum_{2}^{(2)}(10)=2 \quad \sum_{3}^{(2)}(10)=5
$$

and referring to our previous table,

$$
\sum_{0}^{(1)}(500)=223, \quad \sum_{1}^{(1)}(500)=277 .
$$

Then equinumerosity means 


$$
\sum_{0}^{(1)}(n) \sim \sum_{1}^{(1)}(n) \sim \frac{1}{2} n,
$$

while the stronger normality means that

$$
\sum_{t}^{(k)}(n) \sim 2^{-k} n
$$

as $n \rightarrow \infty$ for all $t$ and all $k$.

Note that if one has counted the $k$-tuples $\sum_{t}^{(k)}(n)$, one can obtain the counts of $j$-tuples with $j<k$ simply by addition. Thus

$$
\sum_{0}^{(8)}(n)+\sum_{1}^{(8)}(n)=\sum_{0}^{(7)}(n),
$$

and generally

$$
\sum_{2 l}^{(k)}(n)+\sum_{2 t+1}^{(k)}(n)=\sum_{i}^{(k-1)}(n)
$$

for all $k$ and all $t$.

To test normality we have counted the 256 types of 8 -tuples out to $n=2 \cdot 10^{6}$, and we deduced from these the counts, successively, of 7-tuples, 6-tuples, etc.

3. Computing the Parity Individually or En Masse. That the first two terms of equation (1) are equal is fairly obvious. For the simplest proof of the equality of the second and third terms, see [6]. Together, these equations imply Euler's recurrence: For $n \geqq 1$,

$$
\begin{aligned}
p(n)= & p(n-1)+p(n-2)-p(n-5)-p(n-7) \\
& +\cdots+(-1)^{i+1} p\left(n-e_{i}\right)
\end{aligned}
$$

where $e_{i}=\frac{1}{2} i(3 i \mp 1)$, and where the series breaks off just before $n-e_{i}$ becomes negative. One may thus compute the $a_{n}$ en masse by recurrence using (10) modulo 2. For $n$ large about $\frac{2}{3}(6 n)^{1 / 2}$ terms are needed to compute $a_{n}$ if the previous $a_{n-e_{i}}$ are already known.

But MacMahon [7] found the more efficient recurrences:

$$
\begin{aligned}
& a_{4 n} \equiv a_{n}+a_{n-7}+a_{n-9}+\cdots+a_{n-\alpha_{i}} \text { with } \quad \alpha_{i}=i(8 i \mp 1) \\
& a_{4 n+1} \equiv a_{n}+a_{n-5}+a_{n-11}+\cdots+a_{n-\beta_{i}} \text { with } \beta_{i}=i(8 i \mp 3) \quad(\bmod 2) \text {. } \\
& a_{4 n+3} \equiv a_{n}+a_{n-3}+a_{n-13}+\cdots+a_{n-\gamma_{i}} \text { with } \gamma_{i}=i(8 i \mp 5) \\
& a_{4 n+6} \equiv a_{n}+a_{n-1}+a_{n-15}+\cdots+a_{n-\delta_{i}} \text { with } \delta_{i}=i(8 i \mp 7)
\end{aligned}
$$

(Note that $4 n+2=4(n-1)+6$, but the formulas are neater as given.) We will give a proof of (11) presently. For now, let us note the savings possible.

(1) The number of terms for $a_{n}$ (not $a_{4 n}$ ) with $n$ large is now $\sim \frac{1}{4}(2 n)^{1 / 2}$ so that the use of (11) requires only $\sqrt{ } 3 / 8=0.2165$ as much arithmetic as the use of (10).

(2) To compute $a_{n}$ out to $n=N$ we now need to save the $a_{n}$ only to $n=[N / 4]$, so that only 0.25 as much storage is necessary.

Aside from this more efficient computation en masse, there also arises the possibility of iterating (11), and thus of computing an individual $a_{n}$ with no mass 
storage whatsoever, since each application of (11) reduces the arguments by a factor of 4 . We will discuss this possibility briefly later.

4. MacMahon's Congruences. In [7] MacMahon gave a proof of (11) based upon self-conjugate partitions, and in [8] he used (11) to compute the parities out to $n=1000$. Subsequently, independently, and in effect, but not explicitly, G. N. Watson [9] reproved (11) using theta functions. Still later, H. Gupta [10] gave still another proof, this time using Ramanujan's tau function.

Perhaps the most direct proof, since it involves knowledge of none of these special concepts or functions, is this: Since

$$
\frac{1}{1-x^{n}}=1+x^{n}+x^{2 n}+\cdots \equiv 1-x^{n}+x^{2 n}-\cdots=\frac{1}{1+x^{n}}
$$

we have

$$
\begin{aligned}
\frac{1}{(1-x)\left(1-x^{2}\right)\left(1-x^{3}\right) \cdots} & \equiv \frac{1}{(1-x)\left(1+x^{2}\right)\left(1-x^{3}\right)\left(1+x^{4}\right) \cdots} \\
& =\frac{\left(1-x^{2}\right)}{(1-x)\left(1-x^{4}\right)\left(1-x^{3}\right)\left(1-x^{8}\right) \cdots}
\end{aligned}
$$

Thus

$$
\prod_{n=1}^{\infty} \frac{1}{1-x^{n}} \equiv \prod_{n=1}^{\infty} \frac{1}{1-x^{4 n}} \cdot \prod_{n=1}^{\infty} \frac{1-x^{2 n}}{1-x^{2 n-1}}
$$

Since the product on the right equals $\sum_{n=0}^{\infty} x^{n(n+1) / 2}$ (see [11] for the shortest proof) we have

$$
\sum_{n=0}^{\infty} p(n) x^{n} \equiv \sum_{n=0}^{\infty} p(n) x^{4 n} \sum_{n=0}^{\infty} x^{n(n+1) / 2}
$$

and comparing like powers of $x$, congruences (11) follow quickly.

It may be of interest to indicate the quite extraneous considerations that led us to this problem. One of us was in the process of reviewing [12] The Groups of Order $2^{n}(n \leqq 6)$, by Marshall Hall, Jr. and James K. Senior, Macmillan, New York, 1964. The abelian groups there are designated as belonging to a family $\Gamma_{1}$, and the number of such groups of order $2^{n}$ is, of course, $p(n)$. It may be noted, see pages 103-104, that the lattice diagrams of these groups suggest that they fall into dual pairs. The question of whether $p(n)$ is even or odd is therefore the question of whether there are an even or odd number of lattices which are self-dual.

This leads one to consider self-conjugate partitions and thus to rediscover (11) with (essentially) MacMahon's proof. But the proof above is somewhat simpler. Naturally, after having "discovered" the efficient congruences (11), one is eager to exploit them.

5. Normality. We show in Table 1 the value of $\frac{1}{2} m=.676 \cdots$ in octal to 3200 places. In this one can read $a_{n}$ for $0 \leqq n \leqq 9599$. We have placed in the UMT file of this journal the complete 213-page value of $\frac{1}{2} m$ out to $n=2,039,999$. In Tables 2 and 4 we list the counts of the 8-tuples $\sum_{t}^{(8)}(n)$ for $t=0(1) 255$ and $n=10^{6}$ and $2 \cdot 10^{6}$, respectively. For example, 


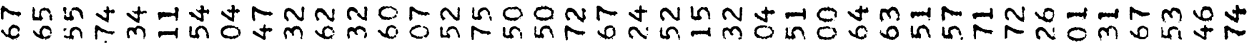

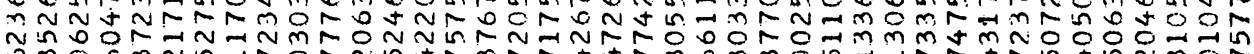

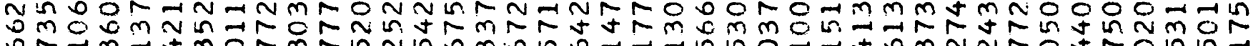
ố

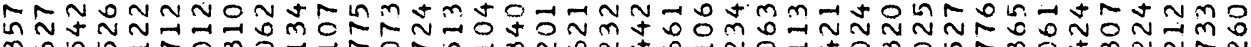
m

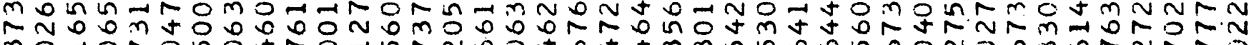

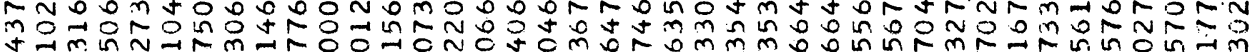
† Nin广ษ

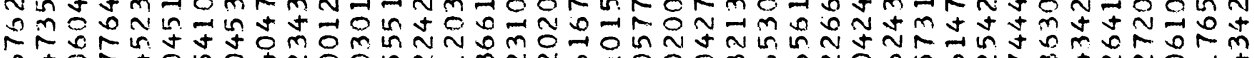

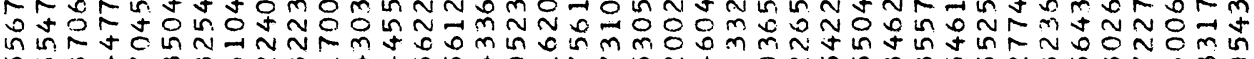
ñ oNom $0 N-N O$ In

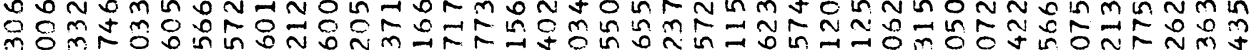

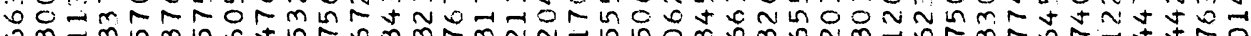
Sm $m$ m $m$ in

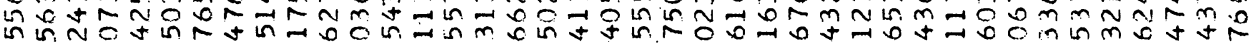
ont

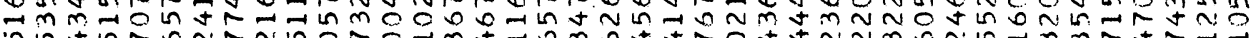

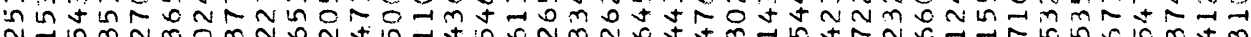

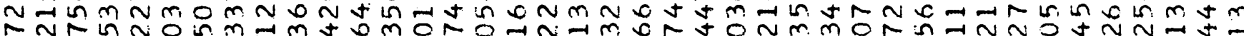
= ntman

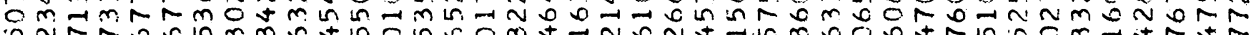

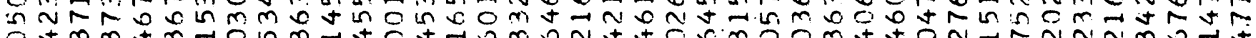

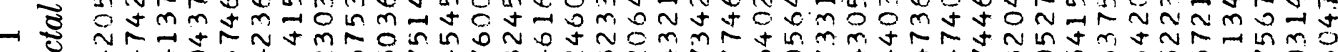

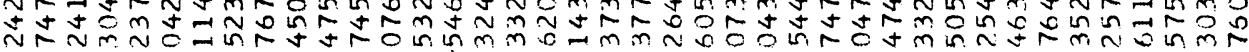

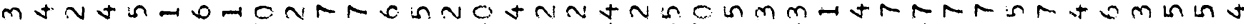
o 0 0 tomj

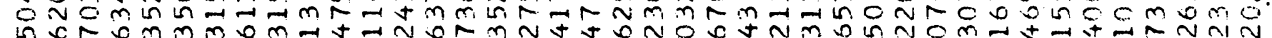
L

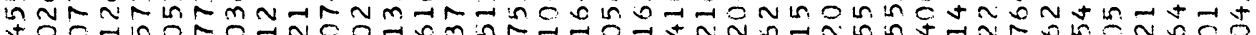
†OO0

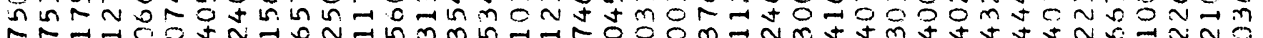
人

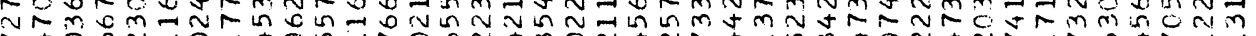

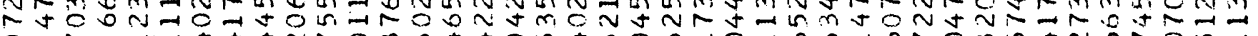

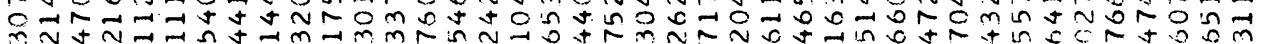

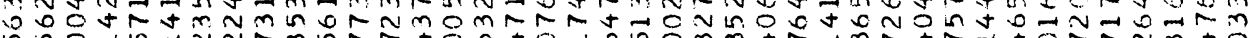
织织$m \Omega$ L

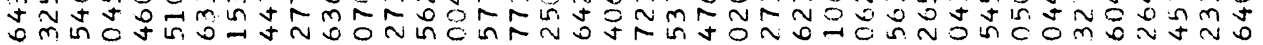

ontmNONDAR

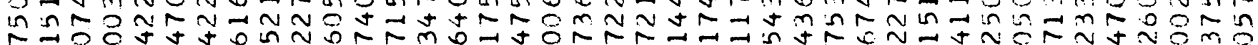

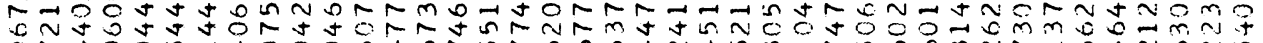
orjo $m m+c 0 N \pi$ L t ningtanmomoton-tant

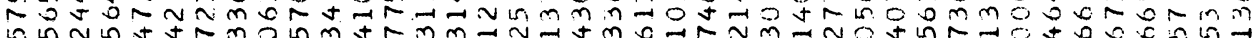

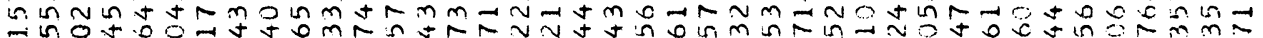

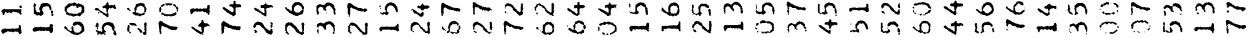

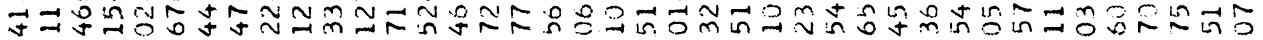

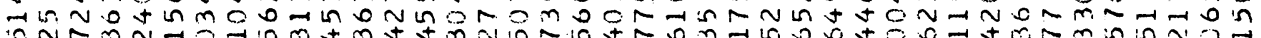

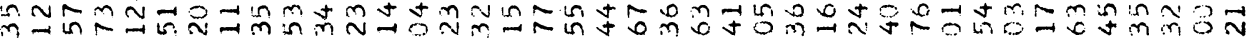

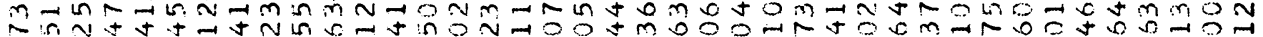

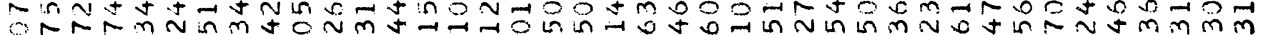
ด๊

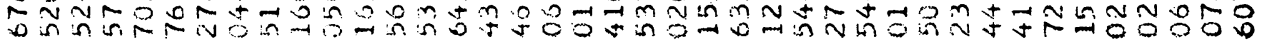




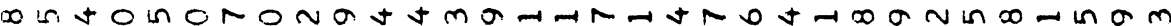

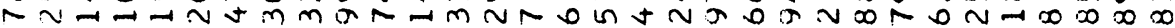

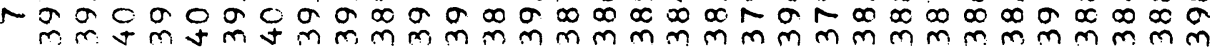

-

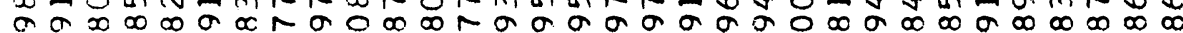
$n m n m m m m m n j m m m m m m m m m m n j m m m m m m m m m m$

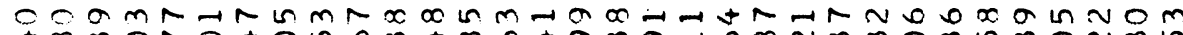

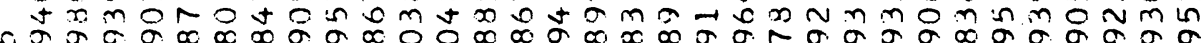
$m m m m m m m m m m$ j $m m m m m m m m n m m m m m m m m m m$

2

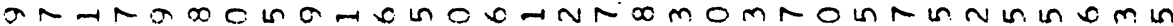

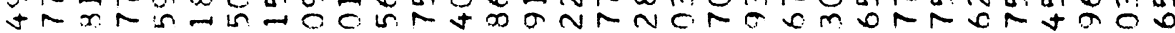
o

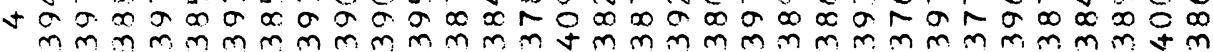

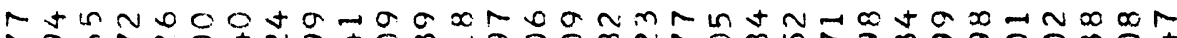

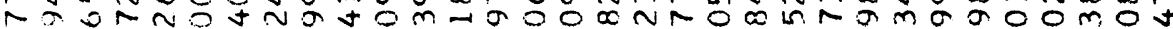

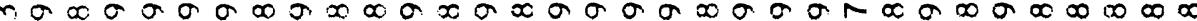
$m m m m m m m m m m n m m m n m m m m m m m m m m m m m m m m m$

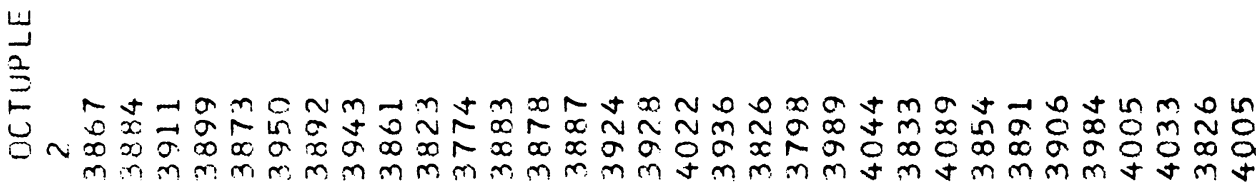

$x$ t

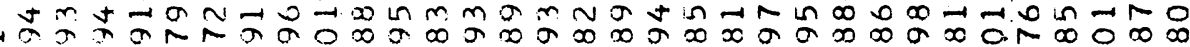

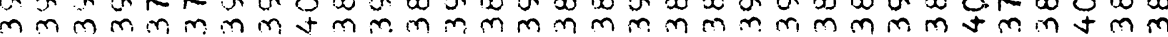

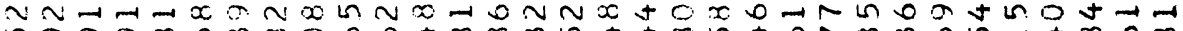

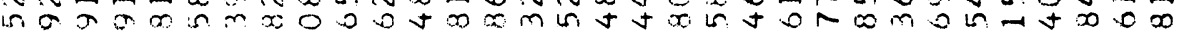

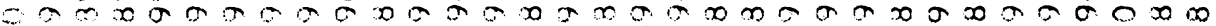
$m n m m m m m m m m m m m m m m m n m m i n$ 
$\cos a \ln a n \rightarrow 0$ a

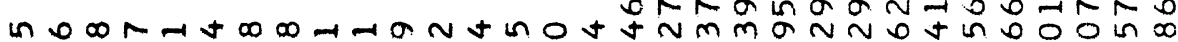

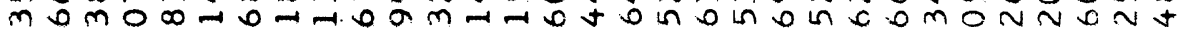

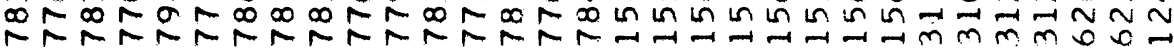

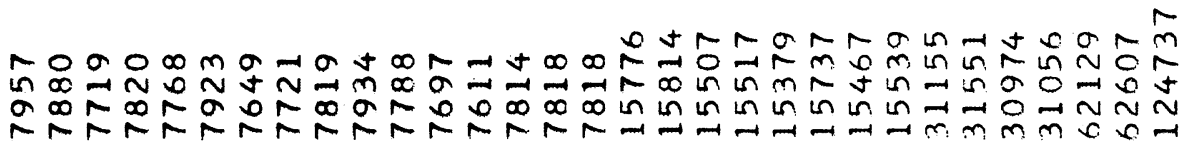

$\sim+$

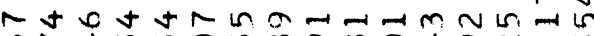

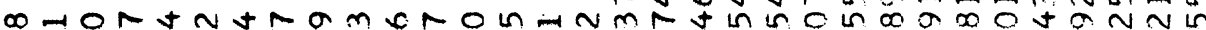

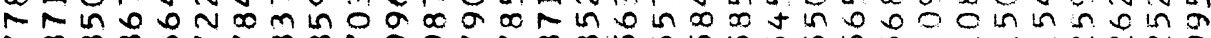

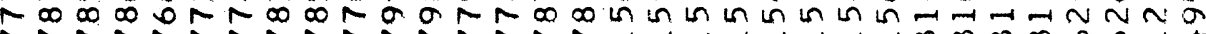
GNRRRRRRRRRRRRRAa

2

a O

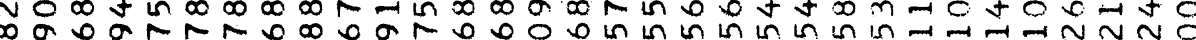
OR 언 늘

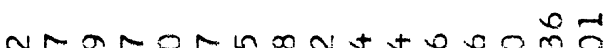

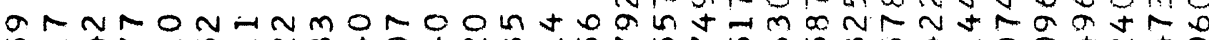

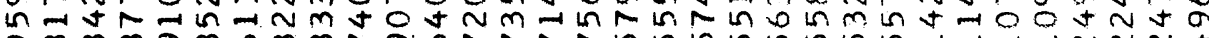

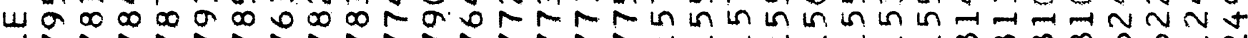

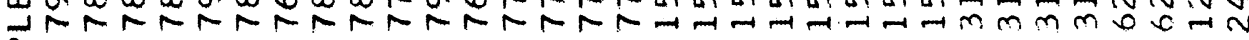
$\frac{2}{1}$

- 0 t t ot O

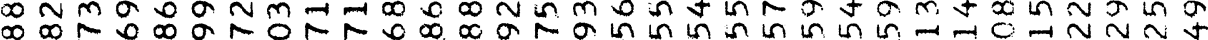

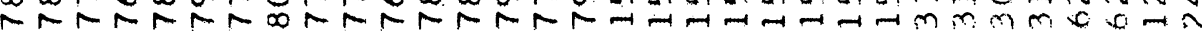

$\infty \sim \ln \pi$

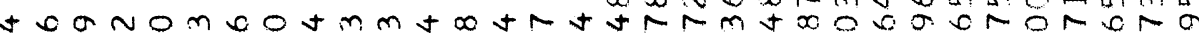

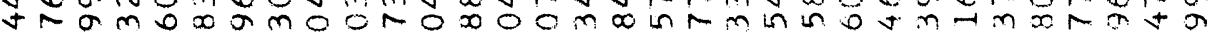

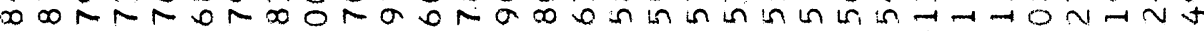

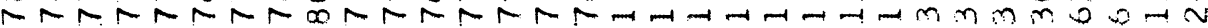
obaranR

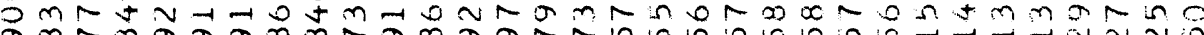

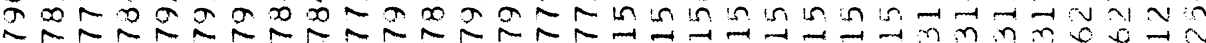




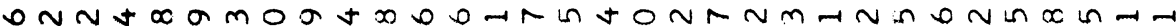

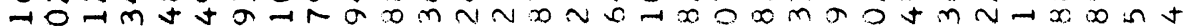

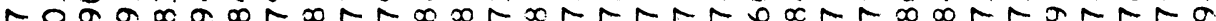

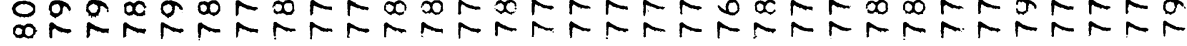

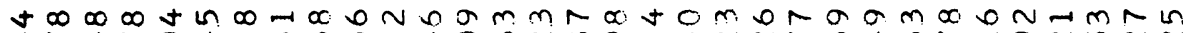
trano

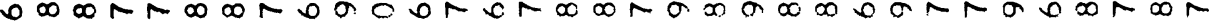

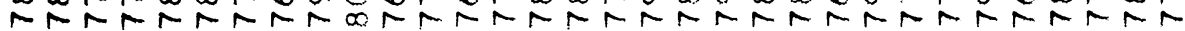

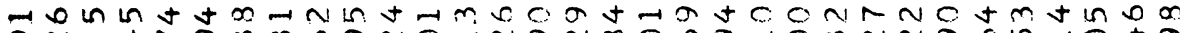

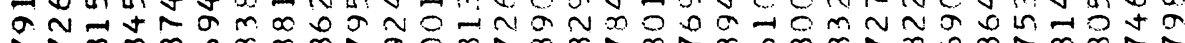

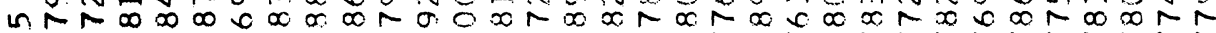

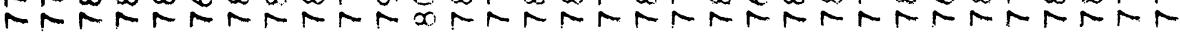

$a$
$a$
$a$
$a$
$a$
$a$

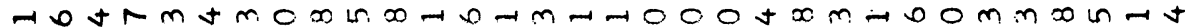

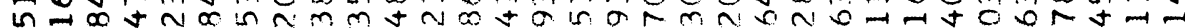
+

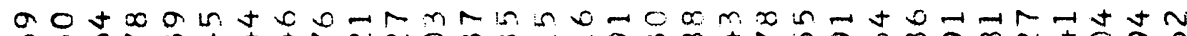

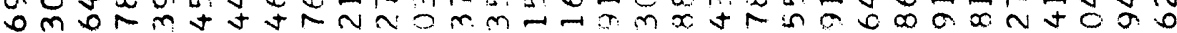

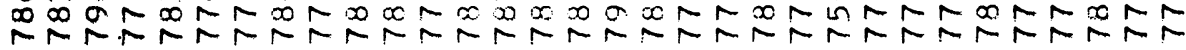

崩

a n

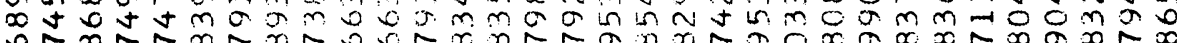

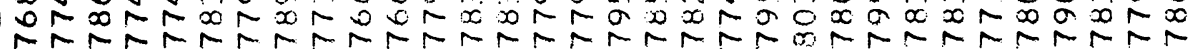

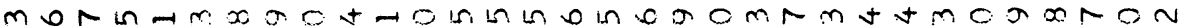

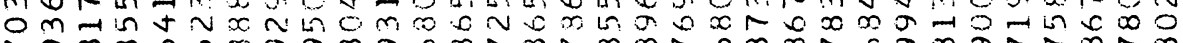

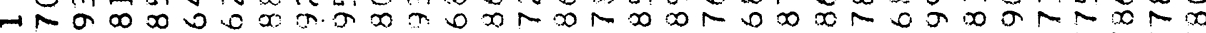

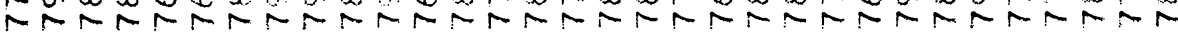

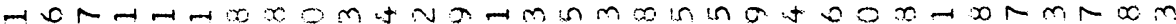

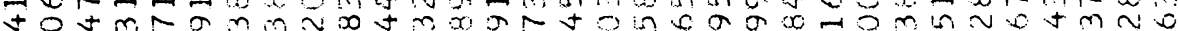

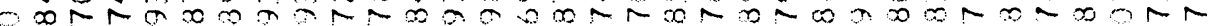

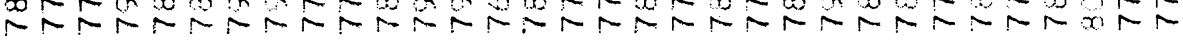




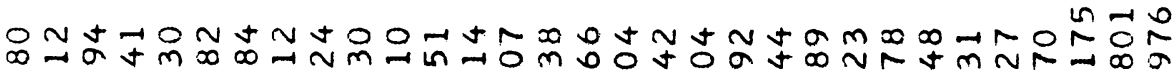

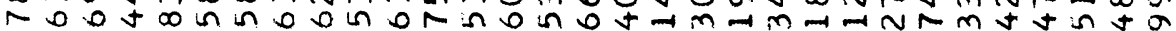

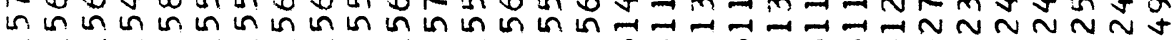

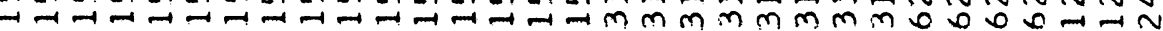

$0 \ln \pi$

NND-ONNO ON

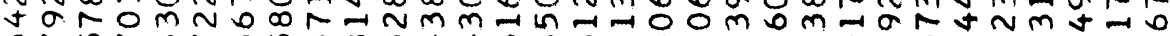

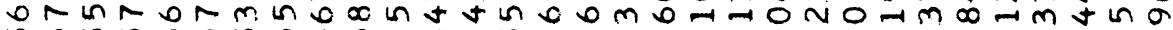

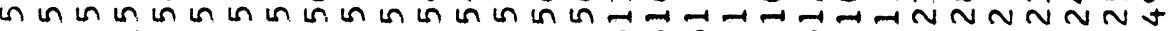

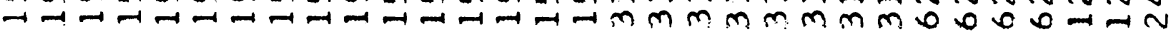

$\sim \ln \sim N$

纸attomtin

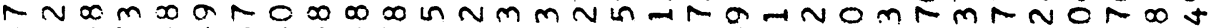

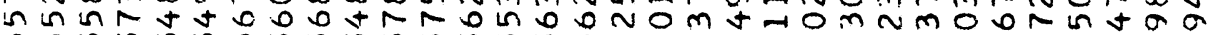

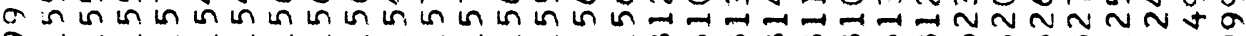

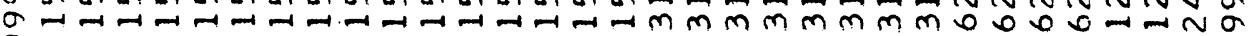

a

a

a

$N \circ \propto m$

NotrNN 0 $0+\infty \rightarrow 0 \infty-1, m$ in or no in ot

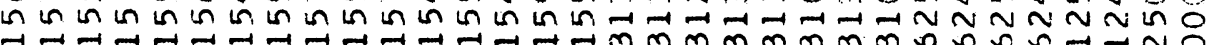

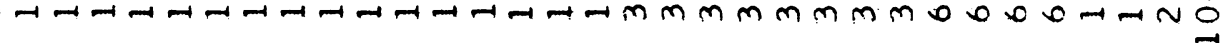

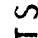

3

$\rightarrow 0 \rightarrow N$

ONHTOLONN

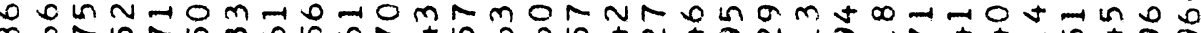

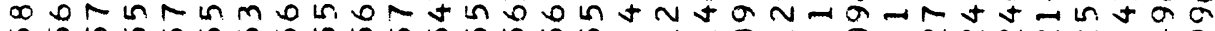

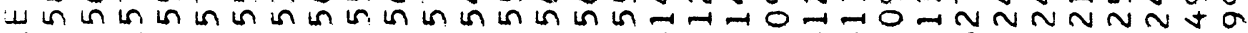

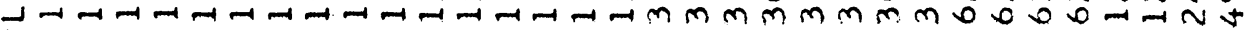
$\frac{2}{3}$

1

$x$

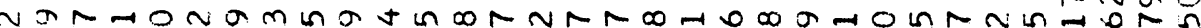
ta a $\sigma$ a

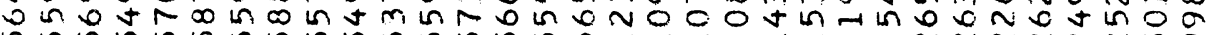

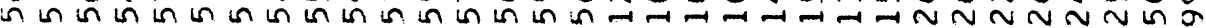

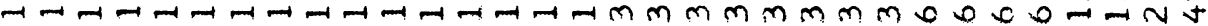

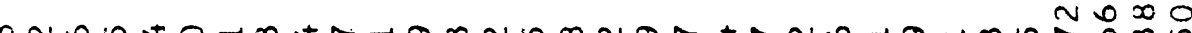
n

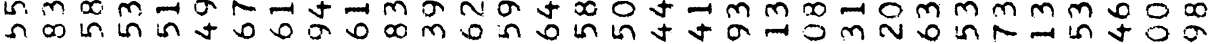

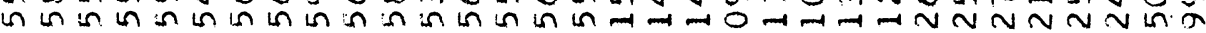

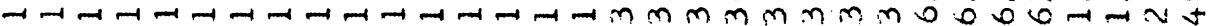

$m \sim$ in $n$

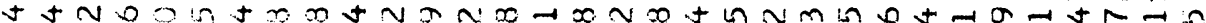

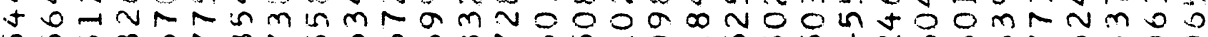

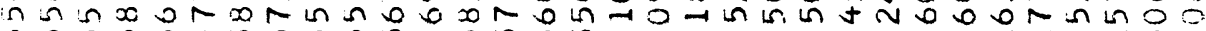

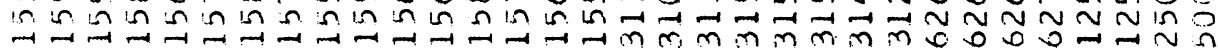




$$
\sum_{0}^{(8)}\left(10^{6}\right)=3952 \text { and } \sum_{12}^{(8)}\left(2 \cdot 10^{6}\right)=7916 .
$$

These tables are read first across, and then down, for increasing $t$.

From Tables 2 and 4 we compute the counts of $k$-tuples for $k=7,6, \ldots, 1$ at $n=10^{6}$ and $2 \cdot 10^{6}$, respectively. This is done by use of the recursion (9), and the results are listed in Tables 3 and 5 in the obvious way. Thus

$$
\sum_{0}^{(7)}\left(10^{6}\right)=7900, \quad \sum_{1}^{(6)}\left(10^{6}\right)=15848, \quad \sum_{2}^{(5)}\left(2 \cdot 10^{6}\right)=62655 .
$$

The initial impression of this data is that no type of $k$-tuple is favored over other types, that the various types are equidistributed, and that the data here is consistent with the hypothesis of normality. We have attempted no elaborate statistical tests of this, but we did examine Good's psi-square serial test [13], [14] to a limited extent. Let

$$
\psi_{k}^{2}=2^{k} n^{-1} \sum_{t=0}^{2^{k}-1}\left(\sum_{t}^{(k)}(n)-2^{-k} n\right)^{2} .
$$

Good showed that if the bits of a binary number are random, then $\psi_{k}^{2}$ has an expectation $2^{k}-1$. We list these $\psi_{k}^{2}$ for $k=1(1) 6$ and $n=10^{6}, 2 \cdot 10^{6}$ together with their expectation in Table 6.

TABLE 6

\begin{tabular}{c|c|c|c}
\hline$k$ & $n=10^{6}$ & $n=2 \cdot 10^{6}$ & Expect. \\
\hline 1 & 0.796 & 0.506 & 1 \\
2 & 1.631 & 1.192 & 3 \\
3 & 7.737 & 2.662 & 7 \\
4 & 23.106 & 9.429 & 15 \\
5 & 44.329 & 21.770 & 31 \\
6 & 87.733 & 56.850 & 63 \\
\hline
\end{tabular}

Now note: We are testing here for randomness, but we are really interested in normality. The former implies the latter, but what of the converse? The data in Table 6 is consistent with randomness, and therefore also with normality. At $n$ $=2 \cdot 10^{6}$ (but not at $n=10^{6}$ ) the distribution is even "too good." It seems to us conceivable (but admittedly, we are now going somewhat beyond our competence) that real numbers may exist with the $\psi_{k}^{2}$ consistently too small. While such behavior would not be random, it could still imply normality-in fact, the smaller the $\psi_{k}^{2}$ are, the better.

6. Equinumerous Evens and Odds. Turning now to $k=1$ in greater detailand the question whether even and odd partition numbers are equinumerous-we list in Table 7 the number of odds, $\sum_{1}^{(1)}(n)$, and the ratio of odds to evens $\sum_{1}^{(1)}(n) / \sum_{0}^{(1)}(n)$ for $n=50,000(50,000) 2 \cdot 10^{6}$.

Since these steps $\Delta n=50,000$ are large and therefore do not allow a completely accurate picture of the variations in the ratio function, we supplement Table 7 with 
the description in Table 8 . This lists 11 regions, A through $\mathrm{K}$, within each of which the ratio remains continually greater than 1 , or continually less than 1 . Thus, the early preponderance of the odds, that we already noted, continues throughout region A until $n=6672$. Between these regions there are many small oscillations of the ratio function around the value 1 . For example, between regions $G$ and $H$, the difference:

$$
\text { odds - evens }
$$

varies between +56 and -65 , and the ratio equals 1 for 176 different values of $n$ (including, as in Table $7, n=400,000$ ).

TABle 7

\begin{tabular}{r|r|c|c|c|c}
\hline$n \cdot 10^{-4}$ & Odds & Ratio & $n \cdot 10^{-4}$ & Odds & Ratio \\
\cline { 2 - 5 } 5 & 25016 & 1.00128 & 105 & 524597 & 0.99847 \\
10 & 50200 & 1.00803 & 110 & 549632 & 0.99866 \\
15 & 75041 & 1.00109 & 115 & 574646 & 0.99877 \\
20 & 99766 & 0.99533 & 120 & 599770 & 0.99923 \\
25 & 124703 & 0.99526 & 125 & 624669 & 0.99894 \\
30 & 149758 & 0.99678 & 130 & 649700 & 0.99908 \\
35 & 175105 & 1.00120 & 135 & 674581 & 0.99876 \\
40 & 200000 & 1.00000 & 140 & 699672 & 0.99906 \\
45 & 225123 & 1.00109 & 145 & 724763 & 0.99935 \\
50 & 250016 & 1.00012 & 150 & 749745 & 0.99932 \\
55 & 274917 & 0.99940 & 155 & 774859 & 0.99964 \\
60 & 299972 & 0.99981 & 160 & 799757 & 0.99939 \\
65 & 324951 & 0.99970 & 165 & 824694 & 0.99926 \\
70 & 349834 & 0.99905 & 170 & 849627 & 0.99912 \\
75 & 374718 & 0.99850 & 175 & 874724 & 0.99937 \\
80 & 399531 & 0.99766 & 180 & 899622 & 0.99916 \\
85 & 424656 & 0.99838 & 185 & 924804 & 0.99958 \\
90 & 449744 & 0.99886 & 190 & 949733 & 0.99944 \\
95 & 474475 & 0.99779 & 195 & 974570 & 0.99911 \\
100 & 499554 & 0.99822 & 200 & 999497 & 0.99899 \\
\hline
\end{tabular}

TABLE 8

\begin{tabular}{c|c|c|c|c}
\hline Region & Limits & Ratio & Extreme $\psi_{1}(n)$ & At $n$ \\
\cline { 1 - 3 } A & $1-6671$ & $>1$ & $+1.996^{*}$ & $1230^{*}$ \\
B & $16287-48781$ & $<1$ & -1.662 & 21017 \\
C & $49185-151211$ & $>1$ & +2.882 & 78823 \\
D & $162951-332867$ & $<1$ & -1.684 & 241706 \\
E & $333373-363347$ & $>1$ & +0.553 & 347684 \\
F & $363769-375013$ & $<1$ & -0.158 & 367246 \\
G & $376961-395293$ & $>1$ & +0.204 & 386259 \\
H & $406565-494241$ & $>1$ & +0.692 & 434150 \\
I & $538051-601509$ & $<1$ & -0.499 & 569769 \\
J & $637169-645423$ & $>1$ & +0.154 & 641119 \\
K & $646475-2040000+$ & $<1$ & -1.165 & 812968 \\
\hline
\end{tabular}

* Only $n>1000$ examined here. 
Consistent with the definition (13) is the designation $\psi_{1}(n)$ for the normalized difference:

$$
\frac{\text { odds }- \text { evens }}{\sqrt{ } n}=\frac{\sum_{1}^{(1)}(n)-\sum_{0}^{(1)}(n)}{\sqrt{ } n}=\psi_{1}(n) .
$$

As in the previous section, our main interest here is not so much in the distribution of $\psi_{1}(n)$ as in its extreme values, and in Table 8 we list the extreme value it takes on in each interval. For instance, in region $\mathrm{B}$, at $n=21017$ there are 10629 evens and 10388 odds for an extreme value

$$
\psi_{1}(21017)=-1.662 .
$$

In regions $\mathrm{E}$ through $\mathrm{J}$ parity is very much equidistributed. The worst normalized difference occurs in region $\mathrm{C}$ at $n=78823$, with 39816 odds and only 39007 evens. (On Table 7, this $n$ lies between the first two entries, and has a ratio $=1.02074$.)

It is reasonable to conjecture that

$$
\psi_{1}(n)=O\left(n^{\epsilon}\right)
$$

for any positive $\epsilon$. If this is true, then we have not merely that the ratio $\rightarrow 1$, but we also know its rate of convergence:

$$
\mid \text { ratio }-1 \mid<a n^{-1 / 2+\epsilon}
$$

for some $a$, and any $\epsilon$.

7. Runs. The data in Section 5 was extended only to 8-tuples. To go beyond would require massive amounts of data, but the following special cases are of some interest. How often should one expect say, 15, and only 15 consecutive odd partition numbers? Since this presumes that the partition numbers immediately prior to such a sequence and immediately subsequent are both even, we are in fact asking for the count of 17 -tuples of type $2\left(2^{15}-1\right)=65534$. As above, the expectation to $n$ $=2 \cdot 10^{6}$ is

$$
\sum_{65534}^{(17)}\left(2 \cdot 10^{6}\right)=2^{-17}\left(2 \cdot 10^{6}\right)=15.26 .
$$

Actually, there are 16 such runs of exactly 15 successive odds - the first run beginning with $p$ (108417), and the sixteenth beginning with $p$ (1936252).

In Table 9 we indicate the number of runs $\geqq 15$ out to $n=2 \cdot 10^{6}$. There are no runs here greater than 20. All of this data seems to be as expected.

Table 9

\begin{tabular}{c|c|c|c}
\hline$k$ & Even Runs & Odd Runs & Expectation \\
\hline 15 & 10 & 16 & 15.3 \\
16 & 7 & 4 & 7.6 \\
17 & 5 & 5 & 3.8 \\
18 & 2 & 4 & 1.9 \\
19 & 2 & 0 & 1.0 \\
20 & 1 & 0 & 0.5 \\
Total & 27 & 29 & 30.1 \\
\hline
\end{tabular}


Curio-collectors may wish to know that the 20 partition numbers

$$
p(n), \quad 1517214 \leqq n \leqq 1517233
$$

are all even, while

$$
p(n), \quad 617995 \leqq n \leqq 618012
$$

constitutes the first sequence of exactly 18 odd partition numbers.

8. Remarks on the Presumed Normality. The last three sections, taken together, do make a good empirical case for normality (modulo 2). We are indebted to Dr. A. O. L. Atkin for a reason why the modulus 2 and also the modulus 3 would be expected to be special for the partition numbers. All known congruence relations for these numbers can be deduced from the so-called modular forms. Entering here in a fundamental way is the linear function

$$
24 m-1
$$

and while this can be divisible by any prime greater than 3,2 and 3 are clearly special. Therefore, Atkin would also expect normality (modulo 3). We have not examined this.

Of course, such considerations are merely suggestive, and, so far, have not led to a proof of normality for either modulus, 2 or 3.

Another aspect of the distinction here between the apparent normality (modulo 2 ) and the distinct nonnormality (modulo 5), as exemplified in (2) and (5), is that one is reminded of the numbers of Wolfgang Schmidt. As is known, he showed [15], [16] that there exist real numbers $x$ normal to one base $r$ without being normal to another $s$. Perhaps we should clarify the difference between the phenomena presently under investigation and Schmidt's phenomena. Given any sequence of integers, $a(n)$, we could construct two different real numbers as in our equations (3) and (4), and they may, as apparently is the case here, be normal to one base while not to another. On the other hand, a Schmidt number $x$ gives rise to two different integer sequences:

$$
a(n)=\left[x r^{n}\right] \text { and } b(n)=\left[x s^{n}\right] .
$$

Finally, we wish to draw the main inference. Some time ago, Professor Freeman Dyson wrote one of us, "Atkin and I were never able to do anything with modulo 2 [for the partition function]." But if the parity is normal, and this is what our investigation strongly suggests, it appears to be a valid inference that "nothing" can be done - "nothing" surely as simple as the congruence (2), or even as profound as the congruence (5). There remains the problem of proving the presumed normality, but no doubt that will be very difficult. Rather more promising is the weaker problem of showing that every $k$-tuple occurs, that is:

$$
\sum_{t}^{(k)}(n)>0 \quad(\text { every } t, k)
$$

for a sufficiently large $n$. Happily, this implies the (only seemingly stronger) result:

$$
\sum_{t}^{(k)}(n) \rightarrow \infty \quad(\text { all } t, k) .
$$


9. Iterated Computation of the Parity; An Unsolved Problem. As we indicated at the end of Section 3, by iterating equations (11) one can determine individual parities independently of any stored table of $a_{n}$ except for

$$
a_{0}=1, \quad a_{2}=0 .
$$

This leads to an unsolved problem of interest. Let us introduce an abbreviated notation; instead of

we write

$$
a_{200} \equiv a_{50}+a_{43}+a_{41}+a_{20}+a_{16}
$$

$$
200=50,43,41,20,16 .
$$

The algorithm is standardized by use of the three rules:

(a) Replace the largest term on the right by its equivalent in (11).

(b) Whenever two repetitions of an argument appear on the right, cancel them both (since their sum is even in any case).

(c) Repeat until 0 or 2 or 0,2 is all that remains on the right. Example:

For 200 one has the sequence:

$$
50,43,41,20,16,11,10,10,7,10,5,5,4,2,1,0,1,1,0 .
$$

Here we have italicized each term replaced by its equivalent, and used boldface for each pair eliminated by cancelling. Thus $p(200) \equiv p(2)=$ even.

In the computation for 200 we listed 19 terms, and cancelled 4 pairs. We define

$$
t(n) \quad \text { and } \quad c(n)
$$

to be these two functions. Thus

$$
t(200)=19, \quad c(200)=4 .
$$

Let us compute these functions for $n=100,200,300,400,500,600$. To do the algorithm efficiently, it is best not to use (11) directly, but, after having decided whether the current term to be replaced is of the form

$$
4 n, 4 n+1,4 n+3, \text { or } 4 n+6,
$$

respectively, we write down $n$, and then subtract according to the differences:

$$
\begin{aligned}
& 7,2,21,4,35,6,49,8 \text {, etc., } \\
& 5,6,15,12,25,18,35,24 \text {, etc., } \\
& 3,10,9,20,15,30,21,40 \text {, etc., or } \\
& 1,14,3,28,5,42,7,56 \text {, etc. }
\end{aligned}
$$

Here is a brief Table 10 .

Table 10

\begin{tabular}{c|c|c}
\hline$n$ & $t(n)$ & $c(n)$ \\
\hline 100 & 11 & 2 \\
200 & 19 & 4 \\
300 & 30 & 9 \\
400 & 38 & 11 \\
500 & 58 & 16 \\
600 & 56 & 17 \\
\hline
\end{tabular}


We raise the questions whether

$$
\begin{aligned}
& t(n)=O(n) ? \\
& c(n)=O(n) ?
\end{aligned}
$$

Clearly, $t(n)$ will generally increase with $n$, but "luck" plays a part; for 400 and 600 there is much cancellation of large terms, while for 500 there is relatively little.

The real point of our query is the question whether the parity of an individual $p(n)$ can be determined in $O(n)$ operations. If one computed such an individual parity by our previous, en masse, table building, technique the computation would require

$$
\int O(\sqrt{ } n) d n=O\left(n^{3 / 2}\right)
$$

operations. We do not know whether (17) is true.

Aerospace Corporation

Los Angeles, California 90045

David Taylor Model Basin

Washington, D. C. 20007

1. O. Kolberg, "Note on the parity of the partition function," Math. Scand., v. 7, 1959, pp. 377-378. (See also [17], [18]) MR 22 \#7995. $142-149$.

2. HansraJ Gupta, "A table of partitions," Proc. London Math. Soc., 2, v. 39, 1935, pp.

3. Hansraj Gupta, "A table of partitions. II," Proc. London Math. Soc., 2, v. 42, 1937, pp. $546-549$.

4. Morris Newman, "Note on partitions modulo 5," Math. Comp., v. 21, 1967, pp. 481-482.

5. A. O. L. AtKin, "Multiplicative congruence properties and density problems for $p(n)$." (To appear.)

6. Daniel Shanks, "A short proof of an identity of Euler," Proc. Amer. Math. Soc., v. 2, 1951, pp. 747-749. MR 13, 321.

7. P. A. MACMAHON, "Note on the parity of the number which enumerates the partitions of a number," Proc. Cambridge Philos. Soc., v. 20, 1921, pp. 281-283.

8. P. A. MACMAHON, "The parity of $p(n)$, the number of partitions of $n$, when $n \leqq 1000$," J. London Math. Soc., v. 1, 1926, pp. 224-225.

9. G. N. Watson, "Two tables of partitions," Proc. London Math. Soc., 2, v. 42, 1937, pp. $550-556$.

10. Hansraj Gupta, "A note on the parity of $p(n), " J$. Indian Math. Soc., v. 10, 1946, pp. 32-33. MR 8, 566.

11. Daniel Shanks, "Two theorems of Gauss," Pacific Jour. Math., v. 8, 1958, pp. 609-612. MR 20 \#5994.

12. Daniel Shanks, Review of The Groups of Order $2^{n}(n \leqq 6)$ in Math. Comp., v. 19, 1965, pp. $335-337$.

13. I. J. Good, "The serial test for sampling numbers and other tests for randomness," Proc. Cambridge Philos. Soc., v. 49, 1953, pp. 276-284. MR 15, 727.

14. I. J. Good, "On the serial test for random sequences," Ann. Math. Statist., v. 28, 1957, pp. 262-264. MR 19, 73.

15. WolfGang Schmidt, "On normal numbers," Pacific J. Math., v. 10, 1960, pp. 661-672. MR 22 \#7994.

16. WolfGang Schmidt, "Über die Normalität von Zahlen zu verschiedenen Basen," Acta Arith., v. 7, 1961/1962, pp. 299-309. MR 25 \#3902.

17. Morris Newman, "Periodicity modulo $m$ and divisibility properties of the partition function," Trans. Amer. Math. Soc., v. 97, 1960, pp. 225-236. MR 22 \#6778.

18. J. H. van Lint, "Solution of problem 4944," Amer. Math. Monthly, v. 69, 1962, p. 175. 